\title{
Empresarios y políticos en la Sevilla de la Restauración: la familia Ybarra
}

\author{
María Sierra Alonso* **
}

Esta comunicación resume parte del trabajo de investigación que estamos realizando como tesis doctoral, dedicado al estudio de la clase política conservadora sevillana de la Restauración. Podemos ofrecer ya un primer adelanto, que en forma de Tesina y con el mismo título que el que encabeza estas páginas, presentamos en la Universidad de Sevilla en julio de 1989. Este trabajo, cuyas conclusiones iniciales proporcionamos, está centrado en el estudio de unos miembros característicos de la clase dirigente provincial, los Ybarra, líderes durante veinticinco años del Partido Conservador sevillano.

\section{METODOLOGIA Y FUENTES}

Gracias a trabajos ya realizados bajo las directrices de un programa de investigación desarrollado en el departamento de Historia Contemporánea de la Universidad de Sevilla por el profesor Sánchez Mantero, conocemos importantes aspectos de la vida política sevillana durante la Restauración. El hecho de que estos trabajos ofreciesen una visión del sistema desde el punto de vista de partidos marginales al mismo, y el de

* Universidad de Sevilla.

** Abreviaturas utilizadas: APFY, Archivo Privado de la Familia Ybarra (Sevilla). HPFY, Hemeroteca Privada de la Familia Ybarra (Sevilla). HMS, Hemeroteca Municipal de Sevilla. 
que estuviesen centrados en los años de su formación y crisis nos llevó a proponer una investigación en torno a las fuerzas sustentadoras del régimen durante sus años centrales.

Desde un primer momento nos pareció necesario el afrontar el estudio de la naturaleza del sistema político de la Restauración desde el análisis de la clase dirigente que lo mantuvo y usufructó: la determinación de la posición concreta de este grupo respecto al resto de la sociedad, y de las consecuencias de este status socio-económico en la vida política, permitirá ofrecer la estructura básica de poder en virtud de la cual se configuraron los modos políticos característicos de la Restauración. El conocimiento de las redes de clientelas, sus patrocinadores, la relación de preeminencia o subordinación de éstos respecto al poder central, el tipo de lazos de dependencia, etc., explicará en parte la existencia de esas determinadas manifestaciones políticas, como el encasillado o los mecanismos de presión electoral, que, aunque se hacian más patentes en los momentos electorales, se debían a una realidad de fondo que funcionaba cotidianamente.

La opción metodológica expuesta se vio reforzada por el hecho de poder acceder a un tipo de documentación que consideramos privilegiada. Se trata del bien conservado archivo de la familia Ybarra, jefes del Partido Conservador y eminentes miembros de la oligarquía económica provincial. La importancia de los archivos privados para el conocimiento de la Restauración está en relación con la naturaleza misma de su sistema político, ya que el predominio de las relaciones privadas en la vida pública de este período hace que el reflejo fundamental de tales comportamientos escape con frecuencia a fuentes de índole pública (como la prensa), y quede limitado al círculo de la correspondencia privada.

La riqueza de los fondos Ybarra referidos a la Restauración se fundamenta en su coherencia cronológica y su variedad documental. Aunque el núcleo del archivo está formado por legajos de correspondencia, también contiene documentos tan interesantes como pactos electorales firmados por los jefes provinciales de los partidos turnantes o recibos de compras de votos. Por otra parte, el Archivo Ybarra no limita su riqueza a la documentación estrictamente privada, ya que incluye una colección de prensa muy interesante, por el doble motivo de ser única y de conservarse bastante completa. Está formada por los distintos periódicos que sucesivamente ejercieron la función de órganos del Partido Conservador sevillano, y ofrece, por tanto, una información inexistente en la prensa liberal y republicana conservada en la Hemeroteca Municipal de Sevilla. 


\section{EL GRUPO FAMILIAR Y LA SOCIEDAD SEVILLANA}

Al proceder a la identificación de los miembros del grupo familiar objeto de estudio constatamos una relativa variedad de individuos, ya que fueron tres generaciones de esta familia las que se sucedieron durante el ámbito cronológico de la Restauración (aunque fue la segunda fundamentalmente la que ocupó este marco temporal).

Pero a pesar de esta multiplicidad de protagonistas, en la Sevilla de estos años se utilizó siempre el apellido Ybarra con un sentido genérico, haciendo referencia a todo un grupo familiar y a su casa comercial. En 1893 un periódico local, al hacer la apología de Eduardo Ybarra como candidato conservador en las elecciones generales, argumenta a su favor el ser «representante de su casa de crédito rica y emprendedora... donde el trabajo continuo de sus dueños les hace acreedores de la estimación pública", o el hecho de "que llevar "el nombre de la Casa Ybarra" es mucho..." 1 .

La actuación conjunta y acorde de todos los miembros de la familia Ybarra, tanto en la vida política como en la gestión de los variados sectores económicos objetos de su inversión, propició la formación de este concepto. El mantenimiento de su patrimonio familiar indiviso en régimen de explotación comunitaria, la intensa comunicación entre los miembros del grupo y la continuación en el tiempo por distintas generaciones de un mismo tipo de actuación política y económica, fueron las bases de esta coherencia de comportamiento que confirió a la familia Ybarra una presencia característica en la vida sevillana de la Restauración.

Por otro lado, los Ybarra, a partir de su asentamiento en Sevilla a mediados del siglo xix procedentes de Bilbao, protagonizaron una rápida y completa imbricación en la clase dirigente provincial. La inclusión en la oligarquía económica sevillana (natural en virtud a su propia relevancia y reforzada por enlaces familiares con la burguesía agraria y ganadera) ${ }^{2}$ se completó con el alistamiento en la clase política local. En este segundo aspecto, la espontánea intervención inicial también se vio respaldada por enlaces y amistades con importantes miembros de la clase política sevillana. Una de estas interesantes relaciones fue la amistad iniciada por el I conde de Ybarra, patriarca de la familia, con el conde de

1 El Universal, HPFY, 2 de marzo de 1893 y 8 de marzo de 1893.

${ }^{2}$ Ybarra Hidalgo, E., Noticias sevillanas de cinco hermanos, Sevilla, 1985. 
Casa Galindo, organizador del Partido Conservador sevillano a comienzos de la Restauración. Esta relación fue continuada por los hijos del I conde de Ybarra, tal como escribe Luis Ybarra a su hermano Tomás tras la muerte de su padre: "El conde de Casa Galindo ha estado hoy a vernos... para darnos el pésame y además decirnos cómo quería él a papá y éste lo distinguía, y quería seguir con nosotros en las buenas relaciones que tenía con papás " ${ }^{3}$ y poco después se fortaleció con un matrimonio entre componentes de ambas familias. El establecimiento de este tipo de enlaces es importante en un sistema político como el de la Restauración, en el que el principal factor de cohesión dentro de los partidos son las relaciones personales, y en el que no es infrecuente que los puestos en los comités se «hereden» en virtud a parentescos familiares ${ }^{4}$.

De esta manera, la participación de la familia Ybarra en la vida sevillana se convirtió rápidamente en un protagonismo que les llevó a ocupar variados puestos directivos y representativos. Entre los más significativos, aunque no aparentemente más importantes, figuran las presidencias de los dos principales casinos locales ${ }^{5}$, ya que estas asociaciones, en su acrisolada simbiosis de relevancia social y preeminencia económica, fueron unas de las más características creaciones de la clase dirigente sevillana ${ }^{6}$.

\section{LA BASE ECONÓMICA DEL PODER POLITICO}

Después de localizar y situar en su marco de actuación a los miembros de la clase dirigente en los que se centra nuestro estudio nos propusimos, como punto de partida indispensable para posteriores profundizaciones, establecer la naturaleza de la base económica de la fami-

${ }^{3}$ Carta de Luis Ybarra a Tomás Ybarra, APFY, 11 de noviembre de 1878.

${ }^{4}$ Carta de A. Maura a Tomás Ybarra, APFY, 18 de julio de 1911.

${ }^{5}$ Eduardo Ybarra presidió el Casino Sevillano de 1909 a 1911, y Tomás Ybarra de 1913 a 1916. La presidencia del Círculo de Labradores fue ocupada por Eduardo Ybarra de 1887 a 1890, y por Tomás Ybarra de 1892 a 1895.

${ }_{6}^{6}$ "L'itinéraire histórique de ce Casino (Círculo de Labradores) est etroitment lié, des sa fondation a la classe dominant de la ville", BERnAL, A. M. y LACROIX, J., "Aspects de la sociabilite andalouse. Les associations sevillanes", Melanges de la Casa de Velázquez, t. XI [1975], pág. 487. 
lia Ybarra. La prioridad de este objetivo viene dada por la evidente y múltiple relación entre el poder político y el poder económico existente durante la Restauración, vinculación que hay que definir con mayor detalle. Con esta idea nos propusimos proceder en primer lugar a la identificación de la fortuna, estudiando los sectores de inversión y su importancia relativa; en segundo lugar al análisis de aspectos reveladores de la mentalidad económica, valorando sobre todo los relacionados con el dinamismo empresarial; y así, finalmente, nos encontraremos en condiciones de definir las relaciones existentes entre la preeminencia económica y el protagonismo político. La determinación del tipo concreto de relación sólo será exactamente aplicable al caso particular analizado, pero permitirá aportar uno de los modelos de relaciones posibles de los múltiples que se dieron, $y$, además, concluir ciertos comportamientos generalizables.

El estudio de los sectores donde los Ybarra distribuyeron su patrimonio y su trabajo perfila un comportamiento económico claramente dinámico. La somera enumeración de los principales sectores de inversión da una idea de ello: fincas rurales, elaboración de productos agrícolas, minas, transportes marítimos, actividades financieras, etc., evidencian una diversificación en la inversión que es, ya de por sí, muestra del notable dinamismo empresarial de esta familia. Pero, además, el análisis y la valoración de estos sectores nos informan de un predominio de los sectores punteros (industria de elaboración alimentaria y transportes marítimos), aunque dentro de una equilibrada combinación con sectores tradicionales (agricultura y ganadería) ${ }^{7}$.

El dinamismo económico de los Ybarra no se manifiesta sólo en el reparto de su inversión, sino, y sobre todo, en la gestión de estos negocios. En este aspecto, hay que destacar tres modos de actuación. En primer lugar, el hábito de combinar una mentalidad abierta a nuevas inversiones con el estudio detallado de las posibilidades de éxito. Un ejemplo de este tipo de comportamiento lo observamos en el proyecto de construcción de una fábrica de abonos, surgido en 1904 con el fin de dar salida a la producción de las propias minas Ybarra. En la correspondencia entre Ramón y Tomás Ybarra queda constancia de esta fructífera combinación de innovación y pragmatismo: «... aunque la cosa no es para dormirse, tampoco urge tanto que no pueda esperar unos días, o

7 Para el conocimiento de los sectores de inversión mencionados, remitimos al trabajo que estamos extractando: SierRA Alonso, María, Empresarios y políticos en la Sevilla de la Restauración: la Familia Ybarra. Sevilla, 1989. 
sea, hasta tener datos ciertos, y mientras tanto yo no dejaré de ir tratando el terreno, pues eso es cosa entretenida, y antes de llegar a contraer un compromiso formal, podremos tener a la vista todos los antecedentes necesarios para resolver lo que nos convenga" 8 .

En segundo lugar, los Ybarra se caracterizaron por una activa función comercializadora, basada en una empresa naviera de ámbito nacional, la "Ybarra y Cia.». Desde tempranas fechas el dinamismo económico de los Ybarra confluyó en estas empresas de comercialización, manifestándose en activas y modélicas aproximaciones a los mercados consumidores. En 1877 por ejemplo, José María Ybarra busca salida para el cobre de sus minas, visitando con este fin a varios industriales siderúrgicos vascos. Así informa a sus hijos del éxito de una visita en Durango: «El cobre que allí tenía, y del que está muy contento, es el de Castillo, y Artaloitia se lo pone a 19 duros el quintal, y a nosotros nos dice ese insustancial que no vende a menos de 21 y 22 , sin duda para que nosotros no bajemos y seguir siendo él exclusivo. Nos dijo que tendría el mayor gusto en volver a gastar del nuestro y le mandamos 10 quintales de prueba, y si le resulta bien gastará 50 mensuales por lo menos. Pepe ha quedado en dar la orden para que afinen el punto de aleación» ${ }^{9}$.

El tercer comportamiento al que antes hacíamos alusión está en directa relación con esta importante actividad comercializadora. Nos referimos al control conseguido por los Ybarra de todas las fases de la cadena de producción en algunos de sus negocios. El caso más evidente es el del aceite: desde la producción de la materia prima en sus fincas hasta la distribución a los mercados consumidores, pasando por la elaboración industrial, los Ybarra controlaron todos los medios de producción (tierras, fábrica y empresa naviera), hecho no muy frecuente ${ }^{10}$. De cualquier manera, el estudio de casos concretos, como el de la familia Ybarra, permitirá superar la tópica disociación entre el propietario agrícola de modos económicos arcaizantes y el industrial-comerciante de modernos métodos.

El comportamiento manifestado por los Ybarra en la gestión de sus negocios revela una mentalidad económica particularmente interesante. En la formación de esta mentalidad jugó un papel esencial la educación

${ }^{8}$ Cartas de Ramón Ybarra a Tomás Ybarra, 28-5-1904 y 2-7-1904. Telegrama de Ramón Ybarra a Tomás Ybarra (1904). APFY.

${ }^{9}$ Carta de José María Ybarra a sus hijos, 22-9-1877. APFY.

10 Ybarra Hidalgo, E., Noticias sevillanas de cinco hermanos. Sevilla, 1985. 
en el trabajo recibida de su padre por los cinco hermanos Ybarra, constatable en la correspondencia entre padres e hijos. La formación en la autoexigencia, la dedicación y el pragmatismo queda patente en estas cartas. En 1876, por ejemplo, les escribe a propósito de ciertos defectos en la contabilidad de la empresa: «Todo esto debiera desaparecer... hacer comprobaciones mensuales por lo menos, y sumar el diario. Es preciso pues recomendar mucho cuidado y vigilar "vosotros mismos" la contabilidad y repasar todos los domingos o todas las noches los asientos de caja, correspondencia y letras del día" ${ }^{11}$.

A su vez, los hermanos Ybarra completaron y transmitieron a sus hijos la formación recibida, cristalizada en un comportamiento económico unitario y coherente. La conservación del patrimonio heredado indiviso y explotado por una empresa familiar ${ }^{12}$ estuvo en la base de tal armonía, y fue, a la vez, factor fundamental del éxito económico. Otras actitudes, como la no delegación de la gestión ${ }^{13} \mathrm{o}$ el control del gasto ${ }^{14}$, consolidaron esta eficaz gestión.

El conocimiento de la base económica de los Ybarra, como el de buena parte de la clase dirigente de la Restauración, es fundamental para comprender las causas de su intervención política. En el caso de la familia Ybarra hemos podido determinar tres factores de naturaleza económica con consecuencias políticas.

El primero de ellos creemos que es generalizable al resto de la clase política, por su relación con la naturaleza misma del sistema de la Restauración. El pacto entre los más fuertes económicamente, para repartirse los beneficios del disfrute alterno del poder mediante un turno pacífico en el Gobierno, está en la base del sistema político de este período, y conllevó un apoyo decidido de la oligarquía económica nacional. Este apoyo directo se reforzó con dos comportamientos: por un lado, la oligarquía económica y política confirió estabilidad al régimen al ampliar el número de beneficiarios del mismo a través del mecanismo del favor, y por otro lado funcionó como agente de conexión entre las realidades locales (cuyos intereses gestionaban en mayor o menor medida) y ese ente lejano que para la «España real» era el Estado.

En este contexto es fácil comprender la importante baza que para la participación política directa era el respaldo de una fortuna personal o

\footnotetext{
1 Carta de José María Ybarra a sus hijos, 15-9-1876. APFY.

12 Testamento de Tomás Ybarra González, 11-11-1916. APFY.

${ }^{13}$ Carta de Eduardo Ybarra a Tomás Ybarra, 3-9-1878. APFY.

${ }^{14}$ Cuentas de gastos de Tomás Ybarra, 1900-1905 y 1912. APFY.
} 
familiar. El disfrute de una base económica notable convertía al poseedor en persona interesada en el éxito del sistema, y, como tal, apta para ejercer cargos públicos. Por otro lado, el disfrute de una fortuna capacitaba a una persona para incluirse en el círculo del mecanismo del favor, tanto a modo de gestionador como de dispensador del mismo. Por ello, éste es uno de los argumentos que la prensa política utiliza a favor de sus candidatos en momentos electorales: en 1893, el órgano de prensa del Partido Conservador, El Universal, argumenta a favor de Eduardo Ybarra, candidato al Congreso, "que el ser condueño de una Casa de Comercio de gran importancia, que el ser labrador y propietario de buenas fincas, que el tener ganadería, que el llevar el nombre de la Casa Ybarra es mucho... Por estas causas cuenta en Sevilla con verdadera influencia» ${ }^{15}$.

La segunda causa económica para la intervención política de los Ybarra es el hecho de verse "naturalmente» impelidos al mundo de la actuación política como consecuencia de la necesidad de defender unos negocios de ámbito nacional y de gestionar medidas favorables para los sectores objeto de su inversión. Así lo expresó Pabón al decir que «la Casa Ybarra actuaba impelida por la necesidad de defender sus intereses de seguras agresiones o como consecuencia de su importancia social que no toleraba inhibiciones» ${ }^{16}$.

Fue, fundamentalmente, el sector naviero el que actuó como obligacio trampolín político. Las causas del protagonismo de este negocio son obvias: primero por ser la inversión más fuerte de todas en las que reparte su dinero la Casa Ybarra, y segundo por ser un negocio de ámbito nacional y alcance público. De esta manera, el hecho de ser principales propietarios de una empresa naviera de tal importancia situó a los Ybarra en el abanico de personas con posibilidades de establecer una correspondencia de favores con líderes políticos nacionales. En 1915, por ejemplo, Dato, entonces presidente del Consejo de Ministros, escribe a Tomás Ybarra solicitándole que la Cía. Ybarra amplíe sus escalas a Portugal para facilitar la exportación de productos españoles, aprovechando la coyuntura favorable de la Primera Guerra Mundial ${ }^{17}$. Lógicamente, Tomás Ybarra se siente capacitado para solicitar, en correspondencia, favores a Dato. En 1916 éste le escribe: "Tendré muy en cuenta los deseos que U. me expresa de que el Sr. Cañal (miembro del Partido Conservador

${ }^{15}$ El Universal, 2-3-1893 y 8-3-1893. HPFY.

${ }^{16}$ PABON, J., Palabras en la oposición. Sevilla, 1939, págs. 244-247.

17 Carta de Eduardo Dato a Tomás Ybarra, 1-11-1915. APFY. 
sevillano) forme parte de la Comisión del Congreso que ha de entender en el proyecto sobre Reglamentación del Trabajo a bordo de los buques mercantes... $" 18$.

Por otra parte, la importancia económica de la familia Ybarra les llevó a figurar en variadas asociaciones económicas sevillanas y a jugar un papel directivo dentro de las mismas. La asunción de tal función directiva les convirtió en representantes de estas asociaciones ante el Estado, en concordancia con su papel de gestionadores naturales de los intereses locales. Es lógico que esta función representativa conllevase una inmediata intervención en política en el desempeño de las gestión de tales intereses, y por ello creemos que el papel directivo asumido por los Ybarra dentro de la oligarquía económica provincial como consecuencia de su propia relevancia es otro factor a tener en cuenta a la hora de calibrar los motivos de su intervención política.

En este sentido, la familia Ybarra al convertirse en representantes de los intereses locales (es decir, los de su oligarquía) actuó como mecanismo de conexión entre el Gobierno de la nación y las microrealidades locales desconocidas desde Madrid. Podemos observar el funcionamiento de este mecanismo de contacto en los momentos en los que la incomprensión entre Gobierno/intereses locales se acentúa: es el caso de las protestas económicas que surgieron en 1899 y 1909 contra el Gobierno Silvela y Maura respectivamente, en sus intentos de aplicar una política económica regeneracionista. Eran reformas interesantes en la teoría pero de muy problemática aplicación, y su puesta en práctica sin más previsiones lesionó intereses económicos antes sólidamente establecidos. En estos casos vemos cómo incluso la oligarquía económica militante en el conservadurismo se levantó contra la nueva política del Gobierno ${ }^{19}$, y Eduardo Ybarra, como jefe provincial del Partido Conservador, tuvo que actuar como intermediario, acomodando la postura del Gobierno con los intereses económicos locales, en una difícil negociación ${ }^{20}$. La ironía de las siguientes palabras de Ramón Ybarra, en carta a su hermano Tomás en 1907 sobre la cuestión catalana, revela lo lejos que la clase económica local se sentía de los políticos profesionales de Madrid: «Por ese camino se prepara una autonomía muy útil, sin duda, para Cataluña, pero perjudicial para el resto del país, que merced a los absurdos aranceles que tenemos, concluirá por convertirse en colonia de Cataluña. Pero, en

${ }^{18}$ Carta de Eduardo Dato a Tomás Ybarra, 18-5-1916. APFY.

${ }^{19}$ Carta de Ramón Ybarra a Tomás Ybarra, (1909). APFY: “Vuestro Silvela no vale para el caso. Aqui hace falta hombre de más coraje aunque no sea tan retórico".

${ }^{20}$ La Monarquía, 21-9-1899. APFY. 
fin, de eso y otras cosas por el estilo deben entender más los políticos madrileños, que tal vez están en el secreto de muchas cosas que en provincias desconocemos" ${ }^{21}$.

\section{EL MECANISMO DEL FAVOR}

La hipótesis, punto de partida de este trabajo, de la importancia del favor personal ( $y$ en general de las relaciones de índole privada) como mecanismo con variadas funciones en la vida pública de la Restauración se vio confirmada a lo largo del estudio. Los archivos privados de la clase política española son la fuente fundamental, y casi exclusiva, para conocer el funcionamiento de este mecanismo. En este sentido, el archivo de la familia Ybarra ofrece una documentación abundante y privilegiada, que, en forma fundamentalmente de correspondencia, nos informa sobre la extensa red de relaciones basada en el favor y la dependencia personal que rodeaba a un político de la Restauración.

La función básica del favor fue la de constituir una «base social» sobre la cual el político pudiese justificar su poder e influencia en una zona determinada de operación. Esta base social se componía de un núcleo central, la clientela, formado por individuos con estrechas relaciones de dependencia, parentesco o amistad con el patrocinador, relaciones originadas por el favor (la concesión de un empleo o auxilio económico, la solución a favor del particular de conflictos con la Administración, etc.) ${ }^{22}$. Este núcleo básico se completaba con la existencia de un amplio grupo de beneficiarios de "favores públicos»: asociaciones económicas, agrupaciones profesionales o la misma ciudad se vieron beneficiadas por gestiones de los patrocinadores ante el poder central a favor de los intereses locales. Los Ybarra se caracterizaron por el ejercicio de esta función: su gestión a favor de los intereses de la provincia que representaban en las Cortes fue intensa, continuada y reconocida por la clase política local. Así, cuando en 1911 un periódico local publica

${ }^{21}$ Carta de Ramón Ybarra a Tomás Ybarra, 19-12-1907. APFY.

22 SiERRA Alonso, Maria: Empresarios y políticos en la Sevilla de la Restauración: la familia Ybarra. Sevilla, 1989. En el Capítulo 3 de este trabajo detallamos la tipología de favores que vinculan a una clientela con su patrocinador, basándonos en la abundante correspodencia entre los Ybarra y distintas personalidades políticas de la Restauración. 
el panegírico de Eduardo Ybarra con motivo de su fallecimiento, afirma: «A la sombra protectora de su influencia vivían infinidad de familias cuya base de existencia la deben al favor o la dádiva unos, y a su caridad inagotables los otros. Siempre tenía la cartera llena de peticiones que se complacía en servir... Cuantos tienen hoy asegurado el pan de sus hijos merced a su influencia o su dinero" ${ }^{23}$.

En la formación de esta doble base social sobre la que los Ybarra apoyaron su intervención política, se empleó tanto el favor dispensado en virtud de la propia capacidad económica como el favor conseguido de la Administración. Esta fecunda mixtificación de comportamientos es lo que nos lleva a afirmar que los Ybarra, como políticos, combinaron caracteres propios de notables económicos de dedicación política secundaria (la concesión del favor por sí mismos) con otros propios de políticos profesionales (la gestión del favor ante la Administración). La estabilidad de este tipo de influencia, y consecuentemente de la clientela que generaba, es mayor que la de un político profesional exclusivamente, que sólo puede recurrir al Estado como fuente de favores para su beneficiados. EI respaldo de una fortuna familiar muy bien invertida permitió a los Ybarra configurarse en dispensadores de favores por sí mismos, no ya como intermediarios ante una autoridad central, sino destino final al que se dirigía una recomendación. La propiedad de la empresa naviera "Ybarra y Cía.", y el poder emplear en ella a un buen número de individuos, les permitió aparecer como dispensadores de favor no sólo ante las personas concretas recomendadas, que pasaban a engrosar la clientela, sino también ante políticos de talla nacional como Dato o Maura, aspecto a tener en cuenta a la hora de calibrar la importancia de la reciprocidad del mecanismo del favor ${ }^{24}$.

Su capacidad económica les permitió otras actuaciones en favor de la ciudad y de la provincia en este mismo sentido: cuando Sevilla sufrió una riada en 1881, la familia Ybarra se ocupó de socorrer a los barrios de San Bernardo y San Nicolás, sustituyendo en estos menesteres al Ayuntamiento, a la vez que efectuaba una importante donación a esta Corporación para ayudas con tal fin ${ }^{25}$.

La red de relaciones sobre la que se apoyaba la actuación de un político de provincias se completaba añadiendo a esta «base social» local

${ }^{23}$ Sevilla, 4-7-1911. HPFY.

${ }^{24}$ Carta de Gómez imaz a Tomás Ybarra, 31-7-1900. APFY. Carta de E. Dato a Tomás Ybarra, APFY, 7-12-1905. APFY. Carta de Maura a Tomás Ybarra, [1913], APFY. Carta de Sánchez de Toca a Tomás Ybarra, [1905], APFY.

${ }^{25}$ El Universal, HPFY, 1-2-1881 y 6-2-1881. APFY. 
el respaldo de la clase dirigente de ámbito nacional, instalada por turno en el Gobierno. Este segundo apoyo se consigue también en buena parte a través del mecanismo del favor: la reciprocidad de favores y recomendaciones solía ser la forma más habitual de conexión entre los políticos locales y los líderes nacionales, como hemos constatado en las relaciones de Ybarra con Maura, Silvela, Dato o La Cierva ${ }^{26}$.

En la vida pública de la Restauración el favor desempeña un segundo papel, ejerciendo una serie de funciones políticas muy concretas, cuyo conocimiento ayuda a profundizar en el de la naturaleza misma del sistema. En primer lugar, hay que hablar del favor como mecanismo electoral en su faceta de instrumento de variada presión. Las relaciones de dependencia basadas en el favor funcionan en todo momento, pero es en los procesos electorales cuando se manifiestan con mayor nitidez, al ser en estas coyunturas políticas cuando se «moviliza» la clase dirigente. El favor en las elecciones puede ser utilizado por su gestionador de diversas maneras: desde el elemental apoyo de la clientela hasta respaldos más importantes por su status social y político. Entre estos últimos figura el de los amigos situados en la Administración de Justicia, quienes colaboraban procesando a miembros de ayuntamientos contrarios o influyendo en causas por delitos electorales. En 1908, por ejemplo, Tomás Ybarra escribe al presidente de la Audiencia de Sevilla: «Mi distinguido amigo: abusando de la reconocida bondad de $U$. me permito recomendar a su atención lo relativo a la causa seguida contra Sanz Sigles y otros por supuestos delitos electorales. Me dicen que el acusador no asistirá a la vista, y yo ruego a $U$. muy encarecidamente vea si es posible acordar su sobreseimiento» ${ }^{27}$.

Pero el apoyo decisivo sobre todo era el de los amigos políticos situados en el poder central, al ser la clase política nacional en el Congreso la que en definitiva decidía sobre la validez o nulidad de las actas electorales. En 1916, por ejemplo, el presidente del Consejo de Ministros, E. Dato, con el que Tomás Ybarra mantenía buenas relaciones, gestionaba recursos electorales siguiendo las indicaciones de éste: «Con todo interés he recomendado el recurso interpuesto contra la nulidad de las elecciones municipales de Bormujos, y celebraría mucho que mi mediación fuese eficaz" ${ }^{28}$. APFY

${ }^{26}$ Cartas de Dato a Tomás Ybarra, APFY, 20-1-1913 y 1-11-1915. APFY.

${ }^{27}$ Carta de Tomás Ybarra al presidente de la Audiencia de Sevilla, F. Pozzi, 4-2-1908.

${ }^{28}$ Carta de Eduardo Dato a Tomás Ybarra, 5-6-4916. APFY. 
En segundo lugar, hemos comprobado cómo el favor operó como una importante base para el liderazgo político, en el contexto de unos partidos de cuadros, sin financiación estructurada y cohesionados por relaciones privadas de amistad y favor entre sus miembros. Desde el cargo de presidente se disponía de la distribución de los puestos en el encasillado (diputados, senadores, diputados provinciales y concejales), y el reparto que el jefe hacia de estos cargos debía respetar compromisos de favores adquiridos y antigüedad en el partido. El acierto en su reparto, agradando al mayor número de miembros posible, era garantía de la estabilidad de una jefatura. Por el contrario, la insatisfacción de los que se consideraban injustamente postergados en el reparto era causa suficiente para justificar el abandono del partido e ingreso en el de la oposición. Este fenómeno se observa en la asunción de la presidencia del Partido Conservador por parte de Eduardo Ybarra: entonces surgió la protesta del elemento del partido autotitulado «de mayor experiencia» contrario al excesivo protagonismo que el nuevo jefe concedia al «elemento joven". En el fondo, la disputa no era sino una excusa para cuestionar un reparto de puestos insatisfactorio ${ }^{29}$.

El favor estaba relacionado con el liderazgo de una manera más general, al convertirse en baremo del éxito de una representación política. La capacidad demostrada por un políico de conseguir beneficios y favores para la localidad que lo elige como representante se considera la medida de su éxito como político profesional, cara a la opinión pública del lugar. Cuando hablamos de "opinión pública" no estamos haciendo referencia a una movilización política mayoritaria, a todas luces inexistente, sino de la opinión de la clase dirigente local, en cuya memoria se archivaba el listado de beneficios conseguidos para la ciudad por los distintos líderes políticos ${ }^{30}$.

Según el éxito en el ejercicio de esta función se configurará uno u otro político como principal figura pública local, a la que hay que acudir en solicitud de su mediación y a la que hay que confiar la representación política. En este contexto hay que entender el pugilato establecido entre los principales líderes políticos locales, el conservador Ybarra y el liberal Borbolla, por figurar como el más efectivo gestionador de los intereses sevillanos ante el poder central, y por tanto como el político más beneficioso para la oligarquía local ${ }^{31}$.

\footnotetext{
${ }^{29}$ La Región, 8-8-1896. HPFY

30 Sevilla, 4-7-1911. HPFY.

31 Sevilla, 12-4-1905, 13-4-1905 y 23-10-1910. HPFY.
} 
Finalmente, creemos que el mecanismo del favor actuó como factor estabilizador del sistema en una doble forma. Por un lado, colaboró a la estabilidad del régimen al extender a un mayor número de sectores sociales los beneficios del mantenimiento del mismo. El círculo de iniciales beneficiarios se ampliaba hacia fuera a través del mecanismo del favor, mediante el cual clientelas y otros beneficiarios eran incluidos en el grupo social de los que disfrutaban del manejo alterno del poder. Las clientelas, por su parte, generaban subclientelas, organizadas en torno a un patrocinador que a su vez dependía de otra cabeza rectora ${ }^{32}$. A través de esta red jerárquica de dependencias los beneficios de la cercanía al poder se extendieron desde el núcleo central hasta los extremos, alcanzando a las microrealidades locales.

La segunda función estabilizadora fue precisamente la conexión que entre esas aisladas realidades locales y el poder central establecieron los políticos locales en su papel de mediadores. La desvinculación entre la Administración estatal y las distintas localidades fue un hecho, expresado en el desconocimiento, la incomprensión y la divergencia de intereses. La función ejercida por políticos locales como Ybarra o Borbolla, mediadores ante el poder central de beneficios y favores para sus pueblos, sirvió para salvar parcialmente tal desconexión. En 1899, por ejemplo, a pesar de las delicadas relaciones políticas de Ybarra con Silvela, Tomás Ybarra visitó a su ministro de Hacienda, Villaverde, para hacer constar "la imposibilidad de que Sevilla pagase las tarifas que, por cédulas persoinales, se le imponen"; y Eduardo Ybarra se ofreció a la cámara de comercio para defender sus intereses, actuación que parece ser que complicó sus relaciones con Silvela ${ }^{33}$.

\section{CONCLUSIONES}

La conclusión más genérica de nuestro trabajo confirma la opción metodológica con la que iniciamos estas páginas: la utilidad de abordar el estudio de la naturaleza de un sistema político desde el análisis de su clase dirigente, y, por ello, la necesidad de determinar la estructura bá-

32 Carta de Luis Zubiria a Tomás Ybarra, 26-3-1915. APFY. Carta de un sobrino a Tomás Ybarra, 31-3-1915. APFY. Carta de una sobrina a Tomás Ybarra, 29-10-1908. APFY.

${ }^{33}$ La Monarquía, 12-7-1899 y 21-9-1899. HPFY. 
sica de poder que este grupo social organiza. Los comportamientos y modos políticos concretos que definen a un sistema político se sustentan sobre esta realidad primaria de poder, y se explican, en buena parte, en función de ella.

El conocimiento de esta realidad debe ser previo al análisis de cualquier sistema político, pero resulta aún más revelador en un régimen como el de la Restauración. El motivo de esta relevancia está vinculado con la primacía de las relaciones privadas entre patrocinadores y clientelas en la vida pública de este periodo. Estas relaciones de dependencia, reciprocidad en el favor y amistad se desarrollaron ante el aislamiento existente entre el ciudadano y el Estado. En el contexto de este marco general, hemos constatado otros fenómenos más concretos, esclarecedores, en nuestro concepto, de la naturaleza del sistema político de la Restauración.

El primero de ellos, por su función básica en la conformación de la estructura del poder, es la evidente y múltiple interrelación entre la preeminencia económica y el protagonismo político. La posesión de un patrimonio importante se convirtió en un apoyo para la intervención política en un doble sentido. En primer lugar, la fortuna de un individuo era la garantía de su idoneidad para el ejercicio de un cargo público, pues su status económico privilegiado le situaba entre los interesados en el mantenimiento del sistema. En segundo lugar, la posibilidad de dispensar favores, basada en la propia fuerza económica, facilitaba la formación de una clientela estable y la inclusión en el "círculo del favor", mecanismo sobre el que se sustentaron buena parte de los modos políticos de la Restauración.

Además de un apoyo, el disfrute de un status económico privilegiado puede convertirse en causa de la intervención política, como sucedió en el caso concreto de la familia Ybarra. Por un lado, la necesidad de defender sus intereses económicos a escala local y nacional funcionó como obligado trampolín hacia la intervención política. Por otro lado, su protagonismo en el marco económico local les llevó a ejercer la función de representantes de la oligarquía económica sevillana a través de variadas asociaciones económicas; la actuación pública inherente a la defensa de estos intereses provinciales derivó en una lógica participación política.

De igual manera que la relación entre la preeminencia económica y el protagonismo político se nos presenta como uno de los fenómenos característicos de la Restauración, existe otro factor esencial para entender la naturaleza de este sistema político: el mecanismo del favor. Esta forma de relación fundamentalmente privada se convirtió en un meca- 
nismo con múltiples funciones públicas: desde el papel más elemental de organizar una red de relaciones que apoye la actuación del patrocinador, hasta el más complejo de servir de estabilizador del sistema, pasando por funciones políticas muy concretas (presión electoral, base de la jefatura de un partido, etc.). La carencia de una red articulada de relaciones entre los ciudadanos y la Administración fue, en definitiva, el contexto en el que se desarrolló este fenómeno, que en el fondo no es sino una trasposición a la vida pública de un modo de relación típicamente privado. Por ello, las relaciones de esta índole, basadas en la dependencia, la amistad o el parentesco, primaron en la vida pública de la Restauración, y esta realidadad está en la base de la explicación de muchos comportamientos políticos característico de este período. 